\title{
Smart Grid Communication Technologies
}

\author{
Dmitry Baimel ${ }^{1}$, Saad Tapuchi ${ }^{1}$, Nina Baimel ${ }^{2}$ \\ ${ }^{1}$ Shamoon College of Engineering, Be'er Sheva, Israel \\ ${ }^{2}$ Sapir Academic College, Hof Ashkelon, Israel \\ Email:dmitrba@sce.ac.il
}

Received 15 May 2016; accepted 30 July 2016; published 2 August 2016

Copyright (C) 2016 by authors and Scientific Research Publishing Inc.

This work is licensed under the Creative Commons Attribution International License (CC BY).

http://creativecommons.org/licenses/by/4.0/

c) (i) Open Access

\begin{abstract}
The smart grid is a new generation of standard power distribution grid. The communication infrastructure is critical for the successful operation of the modern smart grids. The use of communication technologies ensures the reduction of energy consumption, optimal operation of the smart grid and coordination between all smart grids' components from generation to the end users. This paper presents an overview of existing communication technologies such as ZigBee, WLAN, cellular communication, WiMAX, Power Line Communication (PLC), their implementation in smart grids, advantages and disadvantages. Moreover, the paper shows comparison of communication infrastructure between the legacy grid and the smart grid and smart grid communication standards. The paper also presents research challenges and future trends in communication systems for smart grid application.
\end{abstract}

\section{Keywords}

Smart Grid, Communication Technologies, Research Challenges

\section{Introduction}

The legacy grid was built on a principle of unidirectional power flow from the main power plants (that use fossil fuels such as coal, gas and nuclear materials) to the consumers, through the transmission and distribution networks. Due to the increasing electricity demand in the world and the global warming affect, there is a tendency to replace fossil fuels by green renewable energy sources like solar and wind energy [1]-[3]. Furthermore, the legacy grid suffers lack of automated analysis, slow response to quickly changing loading, limited control and poor coordination between generated and consumed energy. This resulted in several major blackouts in the past decades.

The smart grid is the next generation of the power distribution grid that aims to overcome the problems of the legacy grid [4] [5]. Governments of many countries and companies are performing research on smart grid appli- 
cations.

Many technologies that are used in the smart grid are already implemented in other areas of industry such as sensors and wireless networks.

The smart grid uses two-way communications, digital technologies, advanced sensing and computing infrastructure and software abilities in order to provide improved monitoring, protection and optimization of all grids' components including generation, transmission, distribution and consumers.

The smart grid reduces greenhouse gas emissions by application of advanced and controlled large scale integration of renewable energy sources. This large scale integration requires application of advanced distributed control algorithms in order to avoid an unexpected frequency and voltage fluctuations [6]-[8].

By using energy storage systems [9], communication between the grid and customers and advanced algorithms for forecasting generation and loading of the grid [10]-[12], the smart grid ensures full coordination between the generated and consumed energy. This reduces energy losses of the grid, peak demand and energy costs.

Two way communications allows energy consumers to receive accurate real-time prices and bills. The grid operator can receive consumers' real time information about the amount of the consumed energy.

The reliable real-time information flow between all grids' components is essential for smart grid's successful operation. This can be implemented by a reliable and effective communication infrastructure which can be wired or wireless. The advantages of the wireless infrastructure compared to the wired infrastructure are low costs and simple connection to distant and unreachable areas. The disadvantages are interference with other signals and electromagnetic fields and dependence on batteries.

This paper is organized as follows: Section 2 shows comparison of communication infrastructure between the legacy grid and the smart grid; Section 3 presents smart grid communication infrastructure; Section 4 shows overview of communication technologies that can be used in smart grid; Section 5 shows smart grid communication standards; Section 6 presents research challenges, future trends in communication systems for smart grid application; Section 7 presents the conclusions of the paper.

\section{Comparison of Communication Infrastructure between the Legacy Grid and the Smart Grid}

The existing communication infrastructure of the legacy power grid is designed to support only unidirectional power flow from central power plants to the consumers, with limited efficiency and information sharing. The legacy grid communication systems are mainly used for data acquisition from limited number of sensors that are located in the main transmission and distribution points, limited number of control signals transmission and faults detection. The data acquisition is performed by Supervisory Control and Data Acquisition Systems (SCADA) [13] [14].

The smart grid has significantly higher number of sensors and actuators than the legacy grid. They are deployed at all levels of the grid components: Power plants and substation equipment, generators, transformers and home users. The sensors are used for data acquisition and information exchange between equipment and data centers. The actuators are used for optimal control of all grid components. In order to handle such vast data flow, the smart grid must have upgraded, reliable and robust communication infrastructure able to provide real-time secure communications. The communication infrastructure must have wide bandwidth in order to ensure high rate of information flow. Furthermore, the communication infrastructure has to be self-healing and automatically adaptive to changes.

\section{Smart Grid Communication Infrastructure}

The communication infrastructure of the smart grid can be based on three types of networks: Home Area Network (HAN), Neighborhood Area Network (NAN) and Wide Area Network (WAN). The schematic diagram of the smart grid communication infrastructure based on these networks is shown in Figure 1.

HAN is deployed and operated within a small area (tens of meters), usually a house or a small office. The HAN has relatively low transmission data rate compared to other two networks, hundreds of bits per second (bps).

In a typical implementation, a HAN consists of a broadband Internet connection that is shared between multiple users through a wired or wireless modem. It enables the communication and sharing of resources between 


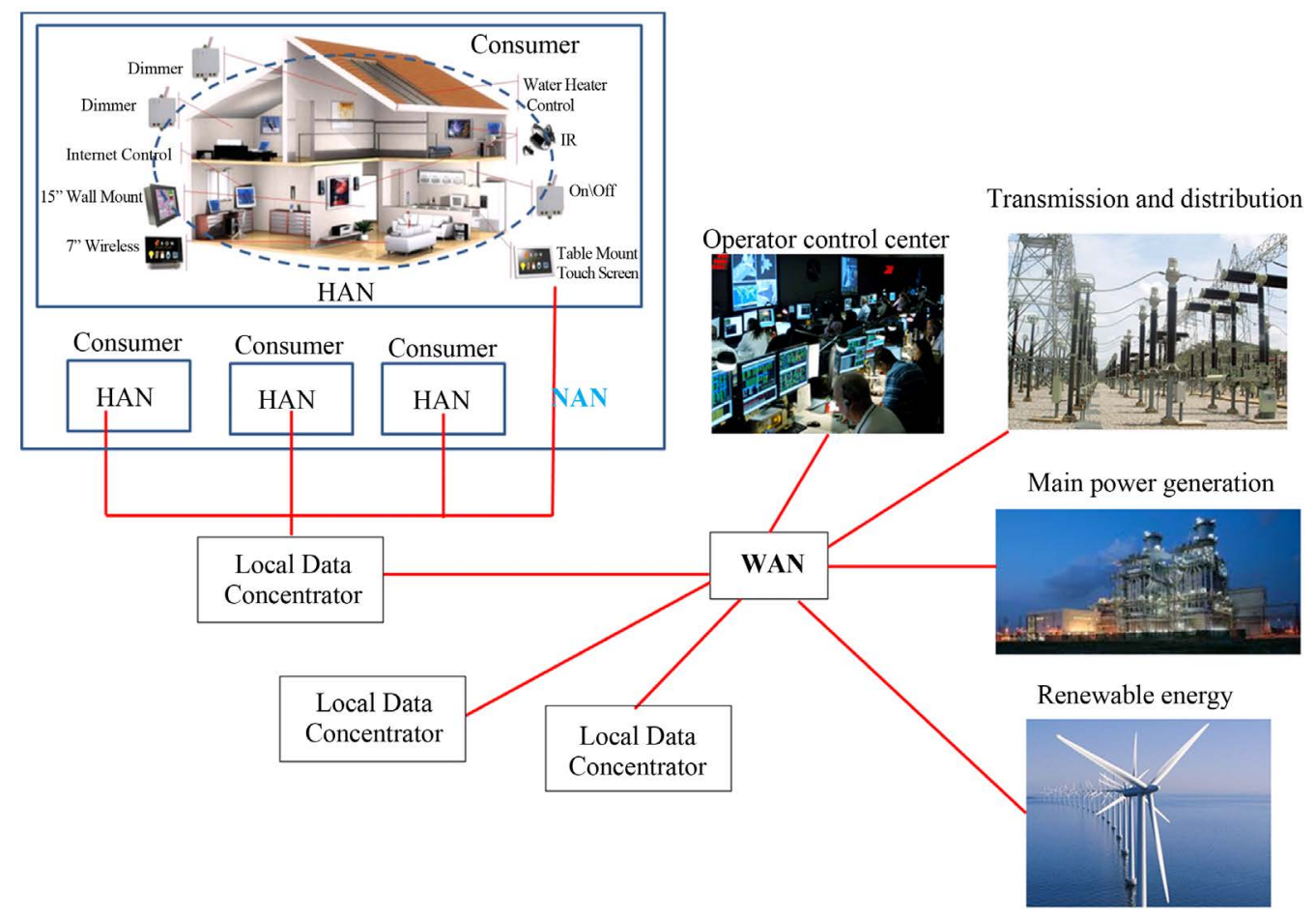

Figure 1. Smart grid communication infrastructure.

computers, mobile and other devices over a network connection. In smart grid implementation, all smart home devices that consume energy and smart meters can be connected to HAN. The devices data is acquired and transmitted through HAN to the smart meters. HAN allows more efficient home energy management. HAN can be implemented by ZigBee or Ethernet technologies.

NAN is deployed and operated within area of hundreds meters which is actually few urban buildings. Several HANs can be connected to one NAN and they transmit data of energy consumed by each house to the NAN network. The NAN network delivers this data to Local Data Centers for storage. This data storage is important for charging the consumers and data analysis for energy generation-demand pattern recognition.

The NAN has up to $2 \mathrm{Kbps}$ transmission data rate. The NAN can be implemented by PLC, Wi-Fi, and cellular technologies.

WAN is deployed and operated within vast area of tens of kilometers and it consists of several NANs and LDCs. Moreover, the communication of all smart grid's components including operator control center, main and renewable energy generation, transmission and distribution, is based on WAN. The WAN has very high transmission data rate up to few Gbps. The WAN can be implemented by Ethernet networks, WiMAX, 3G/LTE and micro-wave transmission.

\section{Overview of Communication Technologies That Can Be Used in Smart Grid}

There are several technologies that can be applied to the smart grid:

\subsection{ZigBee}

ZigBee is based on an IEEE 802.15 standard. ZigBee is used in applications that require a low data rate, long battery life, low cost and secure networking. Applications include wireless light switches, electrical meters with in-home-displays, traffic management systems, and other consumer and industrial equipment that requires shortrange wireless transfer of data at relatively low rates. ZigBee allows connection of up to 60,000 devices to its network.

ZigBee has a defined rate between 20 to $250 \mathrm{kbs}$, best suited for periodic or intermittent data or a single signal transmission from a sensor or input device. The technology defined by the ZigBee specification is intended to be 
simpler and less expensive than other wireless personal area networks (WPANs), such as Bluetooth or Wi-Fi. ZigBee networks are secured by 128 bit symmetric encryption keys.

There is a "ZigBee Smart Energy" application that allows integration of smart meters into the ZigBee network together with other devices [15]. By using this application, smart meters can collect information from the integrated devices and control them. Moreover, the consumers can view their energy consumption in real-time. It also allows better energy consumption and real-time dynamic pricing.

The advantages of ZigBee application in smart grid are low price, small size and it uses relatively small bandwidth.

The disadvantages of the ZigBee are small battery that limits its lifetime, small memory, limited data rate and low processing capability. Moreover, its operation in unlicensed frequency of $868 \mathrm{MHz}$ and $2.4 \mathrm{GHz}$ may have interference with other Wi-Fi, Bluetooth and Microwave signals.

\subsection{WLAN}

A wireless local area network (WLAN) links two or more devices using spread-spectrum or Orthogonal Frequency Division Multiplexing OFDM [16] and usually providing a connection through an access point to the wider Internet. This gives users the ability to move around within a local coverage area and still be connected to the network. Most modern WLANs are based on IEEE 802.11 standards, marketed under the Wi-Fi brand name.

WLANs have become popular in the home due to ease of installation, and in commercial complexes offering wireless access to their customers.

WLAN could be easily integrated into smart grid due to its vast deployment around the world. WLAN works in $2.4 \mathrm{GHZ}-3.5 \mathrm{GHz}$ frequencies.

The advantages of WLAN are low cost, vast deployment around the world, plug and play devices.

The major disadvantage of WLAN is high potential for interference with other devices that communicate on the same frequencies.

\subsection{Cellular Networks}

Cellular networks are largely deployed in most countries and have well-established infrastructure. Moreover, they allow high data rate communications up to 100 Mbps. Therefore, the cellular networks can be used for communication between different components and devices in smart grid. There are several existing technologies for cellular communication such as GSM, GPRS, 2G, 3G, 4G and WiMAX [17]-[22].

The WiMAX technology is the most interesting for smart grid implementation. It is working on 2.5 and 3.5 frequencies, with data exchange rate of $70 \mathrm{Mbs}$ and coverage up to $50 \mathrm{~km}$. The WiMAX chips are integrated inside the smart meters that are deployed through the smart grid.

The advantages of the cellular networks are already existing infrastructure with wide area of deployment, high rates of data transfer, available security algorithms that are already implemented in the cellular communication.

The major disadvantage is that cellular networks are shared with other users and are not fully dedicated to the smart grid communications. This can be serious problem in case of emergency state of the grid.

\subsection{Power Line Communication (PLC)}

Power line communication allows data exchange between devices through electrical power lines. PLC is implemented by adding a modulated carrier signal to the power cables. The data rate of OFDM based communication can be up to several Mbs.

Data rates and distance limits vary widely over many power-line communication standards. Low-frequency (about 100 - $200 \mathrm{kHz}$ ) carriers impressed on high-voltage transmission lines may carry one or two analog voice circuits, or telemetry and control circuits with an equivalent data rate of a few hundred bits per second; however, these circuits may be many miles long. Higher data rates generally imply shorter ranges.

Different types of powerline communications use different frequency bands. Since the power distribution system was originally intended for transmission of AC power at typical frequencies of 50 or $60 \mathrm{~Hz}$, power wire circuits have only a limited ability to carry higher frequencies. It is problematic to establish high frequency communication through power lines due to the dilution of high frequency signals. The propagation problem is a limiting factor for power line communications. 
Furthermore, there can be interference of communication signals with high order harmonics produced by non-linear loads that present in the grid such as rectifiers, inverters, etc. In order to overcome this problem, advanced active filters should be used.

In smart grid applications, the PLC is used in Neighborhood Area Network communication for connecting between smart meters and Local Data Concentrator (LDC). However, the Wide Area Network communication, from LDC to other smart grid components such as operator control center, generation, transmission and distribution, is performed through cellular networks.

The advantage of the PLC is already established, wide-spread infrastructure that reduces installation costs. The disadvantages are presence of higher harmonics in the power lines that interfere with communication signals and limited frequency of communication.

\section{Smart Grid Communication Standards}

The research on smart grid communication technology and protocols is conducted simultaneously in many companies and countries. It is very important to ensure integration of different smart meters, communication protocols and infrastructures. This could be done by development of global standards that will be accepted by all companies and bodies that are involved in the smart grid development worldwide.

There are several authorized organizations that are working on smart grid standardization such as Institute of Electrical and Electronics Engineers (IEEE), European Committee for Standardization, American National Standards Institute (ANSI), International Telecommunication Union (ITU) and more. The most common standards for smart grid communication are shown below. There also additional standards for smart grid communication such as 802.15.4, ISO 1802, IPv4, DNP3, IEC61970, etc.

\subsection{IEEE P2030}

Guide for Smart Grid Interoperability of Energy Technology and Information Technology operation with the Electric Power System and End-Use Applications and Loads. This standard provides guidelines in understanding and defining smart grid interoperability of the electric power system with end-use applications and loads. Integration of energy technology and information and communications technology is necessary to achieve seamless operation for electric generation, delivery, and end-use benefits to permit two way power flow with communication and control. Interconnection and intra-facing frameworks and strategies with design definitions are addressed in this standard, providing guidance in expanding the current knowledge base. This expanded knowledge base is needed as a key element in grid architectural designs and operation to promote a more reliable and flexible electric power system.

\subsection{IEEE P1901}

The IEEE 1901 is a standard for high speed (up to $500 \mathrm{Mbit} / \mathrm{s}$ at the physical layer) communication devices via electric power lines, often called broadband over power lines (BPL). The standard uses transmission frequencies below $100 \mathrm{MHz}$. This standard is usable by all classes of BPL devices, including BPL devices used for the connection to Internet access services as well as BPL devices used within buildings for local area networks, smart energy applications, transportation platforms (vehicle), and other data distribution applications.

IEEE 1901 has been widely recognized as the standard that will enable universal communications in smart grid applications.

\subsection{IEC 62351}

This standard deals with cyber security issues of the smart grid. The different security objectives include authentication of data transfer through digital signatures, ensuring only authenticated access, prevention of eavesdropping, prevention of playback, and intrusion detection.

\subsection{IEC 62056}

EC 62056 is a set of standards for electricity metering data exchange. It includes the following standards:

IEC 62056-42: Physical layer services and procedures for connection-oriented asynchronous data exchange. 
IEC 62056-46: Data link layer using HDLC protocol.

IEC 62056-47: COSEM transport layers for IPv4 networks.

IEC 62056-53: COSEM Application layer.

IEC 62056-61: Object identification system.

IEC 62056-62: Interface classes.

\subsection{PLC G3}

PLC G3 supports high-speed, highly reliable IP-based communications across existing power lines, allowing data and control messages to flow across the generation, transmission, and distribution systems that comprise a regional Smart Grid. It was developed to provide robust connections between smart grid elements to allow the application of advanced billing and demand management techniques to customer loads and to efficiently integrate conventional and renewable-based distributed energy resources, including solar or wind farms.

\section{Research Challenges, Future Trends in Communication Systems for Smart Grid Application}

The smart grid communication system is a complex system that consists of several major subsystems such as smart meters that also operate as transmitter/receiver, wired or wireless communication channels and software based control systems. This complex system has several problems that need to be solved in order to establish reliable and robust communication system. These problems are discussed below:

\subsection{Interference}

As it was mentioned in section III, the basic layer of smart grid communication is Home Area Network. Usually, HAN networks are densely deployed in the urban areas. This dense deployment can cause interference between the HAN networks and result in transmission of unreliable signals from smart meters.

Additional problem is interference inside the HAN network. The future home will have dozens electrical devices that will communicate to the main smart meter. This communication has a great potential to inter home interference. These problems could be solved by developing algorithms that will eliminate/reduce this interference.

There is also interference in the power lines caused by harmonics. This interference could be reduced by using active filters.

\subsection{Data Transmission Rate}

The communication system in smart grid is very important for data acquisition, data analysis and control of the smart grid's components and devices. There thousands smart meters and controlled devices that deployed throughout the smart grid. As a result, there is a huge amount of data that has to be continuously and bi-directionally transferred by the communication system. Therefore, research efforts should concentrate on developing appropriate high data rate communication technology or improving existing technologies such as WLAN or cellular networks. Today, the data rate of WLAN and cellular networks is relatively high compared to other technologies but it should be further increased by using new modulation techniques and improved transmitters/receivers.

\subsection{Standardization}

It seems that in the future smart grid the communication system will be composed from different communication technologies that will be combined together.

Although there are many standards for smart grid communication, there is no sufficient standard and model for integration of different communication technologies in one system. Therefore, such standards should be developed.

\subsection{Cyber Security}

Large amount of data is transmitted through the communication system. This data can be cyber attacked by 
hackers or terrorists. The communication system becomes more vulnerable when it is not fully dedicated to the smart grid communication and shared with other user, e.g. WLAN or cellular network.

The data transmitted in smart grid can be divided into three major groups:

- Measured parameters from smart meters that transmitted to the LDC and to the operator center. By intercepting this data, the hackers can distort the information about the amount of energy consumed by the customers distort billing system and cause economic damage to the electrical company.

- Measured parameters that transmitted to the operator center from smart devices such as switches, transformers, Flexible AC Transmission (FACT) devices, generators, turbines, etc. By intercepting and replacing these signals, hackers can provide wrong information about the equipment and system state and as a result, to cause operator center to make wrong decisions and actions.

- Control signals from the operator center that transmitted to smart devices that were mentioned earlier. By intercepting and replacing these signals, hackers can cause damage to equipment, loose of control of the operator center and major blackouts.

In order to avoid this, the cyber protection has to be applied in both physical and software level. At the physical level, the smart meters and LDCs have to be physically secured to prevent unauthorized access. At the software level, new advanced encryption algorithms has to be developed and applied.

\section{Conclusions}

The future smart grid is based on combination of legacy grid with advanced smart metering, remote sensing, remote control of all key components and equipment. The success of the smart grid depends directly on reliable, robust and secure communication system with high data rate capability.

This paper presented comparison between legacy and future smart grid communication system and an overview of existing communication infrastructure and technologies that can be used for smart grid. The paper also outlined the problems of these technologies and the research challenges that aim to solve these problems.

Future work should concentrate on development of improved security algorithms that could be adapted for the smart grid communication and protocols and methods for interference reduction and elimination.

\section{References}

[1] Xia, X.T. and Xia, J.Z. (2010) Evaluation of Potential for Developing Renewable Sources of Energy to Facilitate Development in Developing Countries. Asia Pacific Power and Energy Engineering Conference (APPEEC), Chengdu, 28-31 March 2010, 1-3.

[2] Zhang, P., Xiao, W. and Choudhury, P. (2011) Communication Systems for Grid Integration of Renewable Energy Resources. IEEE Network, 25, 22-29.

[3] Abbott, D. (2010) Keeping the Energy Debate Clean: How Do We Supply the World's Energy Needs. Proceedings of the IEEE, 98, 42-66. http://dx.doi.org/10.1109/JPROC.2009.2035162

[4] Farhangi, H. (2010) The Path of the Smart Grid. IEEE Power and Energy Magazine, 8, 18-28. http://dx.doi.org/10.1109/MPE.2009.934876

[5] Amin, S.M. and Wollenberg, B.F. (2005) Toward a Smart Grid: Power Delivery for the 21st Century. IEEE Power and Energy Magazine, 3, 34-41. http://dx.doi.org/10.1109/MPAE.2005.1507024

[6] Carrasco, J.M., Franquelo, L.G., Bialasiewisc, J.T. and Galvan, E. (2006) Power-Electronic Systems for the Grid Integration of Renewable Energy Sources: A Survey. IEEE Transactions on Industrial Electronics, 53, 1002-1016. http://dx.doi.org/10.1109/TIE.2006.878356

[7] Grubb, M.J. (2003) The Integration of Renewable Electricity Sources. Elsevier Energy Policy, 19, 670-688.

[8] Lund, H. (2007) Renewable Energy Strategies for Sustainable Development. Elsevier Energy, 32, 912-919. http://dx.doi.org/10.1016/j.energy.2006.10.017

[9] Ibrahim, H., Linca, A. and Perron, J. (2008) Energy Storage Systems-Characteristics and Comparisons. Elsevier Renewable and Sustainable Energy Reviews, 12, 1221-1250. http://dx.doi.org/10.1016/j.rser.2007.01.023

[10] Pai, P. and Hong, W. (2005) Forecasting Regional Electricity Load Based on Recurrent Support Vector Machines with Genetic Algorithms. Elsevier Electric Power System Research, 74, 417-425. http://dx.doi.org/10.1016/j.epsr.2005.01.006

[11] Azadeh, A., Ghaderi, S.F. and Sohrabkhani, S. (2008) A Simulated-Based Neural Network Algorithm for Forecasting Electrical Energy Consumption in Iran. Elsevier Energy Policy, 36, 2637-2644. 
http://dx.doi.org/10.1016/j.enpol.2008.02.035

[12] Azadeh, A. and Tarverdian, S. (2007) Integration of Genetic Algorithm, Computer Simulation and Design of Experiments for Forecasting Electrical Energy Consumption. Elsevier Energy Policy, 35, 5229-5241. http://dx.doi.org/10.1016/j.enpol.2007.04.020

[13] Boyer, S.A. (2009) Scada: Supervisory Control and Data Acquisition. International Society of Automation, USA,

[14] Bailey, D. and Wright, E. (2003) Practical Scada for Industry. Elsevier Linacre House, Jordan Hill, Oxford, UK.

[15] Luan, S.W., Teng, J.H., Chan, S.Y. and Hwang, L.C. (2009) Development of a Smart Power Meter for AMI Based on ZigBee Communication. International Conference on Power Electronics and Drive Systems (PEDS), Taipei, 2-5 November 2009, 661-665. http://dx.doi.org/10.1109/PEDS.2009.5385726

[16] Van Lee, R. and Pramsad, R. (2000) OFDM for Wireless Multimedia Communications. Artech House, Inc., Norwood.

[17] Redl, M., Oliphant, W. and Webber, M.K. (1995) An Introduction to GSM. Artech House, Boston.

[18] Halonen, T., Romero, J. and Melero, J. (2003) GSM, GPRS and Edge Performance Evolution towards 3G/UMTS. 2nd Edition, John Wiley \& Sons, Chichester. http://dx.doi.org/10.1002/0470866969

[19] Garg, V.K. (2001) Wireless Network Evolution: 2G to 3G. Prentice Hall PTR, Upper Saddle River.

[20] Mishra, A. (2004) Fundamentals of Cellular Network Planning and Optimization 2G/2.5G/3G, 4G. John Wiley \& Sons, Chichester. http://dx.doi.org/10.1002/0470862696

[21] Vashney, U. and Radhika, J. (2002) Issues in Emerging 4G Wireless Networks. IEEE Computer, 34, 94-96.

[22] Nuaymi, L. (2007) WiMAX Technology for Broadband Wireless Access. John Wiley \& Sons, Chichester.

\section{Submit or recommend next manuscript to SCIRP and we will provide best service for you:}

Accepting pre-submission inquiries through Email, Facebook, LinkedIn, Twitter, etc. A wide selection of journals (inclusive of 9 subjects, more than 200 journals)

Providing 24-hour high-quality service

User-friendly online submission system

Fair and swift peer-review system

Efficient typesetting and proofreading procedure

Display of the result of downloads and visits, as well as the number of cited articles

Maximum dissemination of your research work

Submit your manuscript at: http://papersubmission.scirp.org/ 